\title{
Quiescent and Active Tear Protein Profiles to Predict Vernal Keratoconjunctivitis Reactivation
}

\author{
Alessandra Micera, ${ }^{1}$ Antonio Di Zazzo, ${ }^{1}$ Graziana Esposito, ${ }^{1}$ Roberto Sgrulletta, ${ }^{2}$ \\ Virginia L. Calder, ${ }^{3}$ and Stefano Bonini ${ }^{2}$ \\ ${ }^{1}$ IRCCS-G.B. Bietti Foundation, 00100 Rome, Italy \\ ${ }^{2}$ Department of Ophthalmology, Campus Bio-Medico University, 00128 Rome, Italy \\ ${ }^{3} U C L$ Institute of Ophthalmology, London EC1V 9EL, UK \\ Correspondence should be addressed to Stefano Bonini; s.bonini@unicampus.it
}

Received 16 November 2015; Accepted 17 January 2016

Academic Editor: Sung-Hoon Kim

Copyright (C) 2016 Alessandra Micera et al. This is an open access article distributed under the Creative Commons Attribution License, which permits unrestricted use, distribution, and reproduction in any medium, provided the original work is properly cited.

Objective. Vernal keratoconjunctivitis (VKC) is a chronic recurrent bilateral inflammation of the conjunctiva associated with atopy. Several inflammatory and tissue remodeling factors contribute to VKC disease. The aim is to provide a chip-based protein analysis in tears from patients suffering from quiescent or active VKC. Methods. This study cohort included 16 consecutive patients with VKC and 10 controls. Participants were subjected to clinical assessment of ocular surface and tear sampling. Total protein quantification, total protein sketch, and protein array (sixty protein candidates) were evaluated. Results. An overall increased Fluorescent Intensity expression was observed in VKC arrays. Particularly, IL1 $\beta$, IL15, IL21, Eotaxin2, TACE, MIP1 $\alpha$, MIP3 $\alpha$, NCAM1, ICAM2, $\beta$ NGF, NT4, BDNF, $\beta$ FGF, SCF, MMP1, and MMP2 were increased in quiescent VKC. Of those candidates, only IL1 $\beta$, IL15, IL21, $\beta$ NGF, SCF, MMP2, Eotaxin2, TACE, MIP1 $\alpha$, MIP3 $\alpha$, NCAM1, and ICAM2 were increased in both active and quiescent VKC. Finally, NT4, $\beta$ FGF, and MMP1 were highly increased in active VKC. Conclusion. A distinct "protein tear-print" characterizes VKC activity, confirming some previously reported factors and highlighting some new candidates common to quiescent and active states. Those candidates expressed in quiescent VKC might be considered as predictive indicators of VKC reactivation and/or exacerbation outof-season.

\section{Introduction}

Vernal keratoconjunctivitis (VKC) is a multifactorial eye disease associated with atopy, characterized by a chronic recurrent bilateral inflammation of the conjunctiva [1]. This childhood disease resolves spontaneously at puberty, although complications might occur due to severe and/or long-standing inflammation, leading to fibrovascular reaction, new collagen deposition, huge tissue remodeling, and permanent visual changes [2]. Recurrent local inflammation might also trigger corneal impairment as well as undesired corneal ulcers [2]. A late onset VKC-like disease has been also observed in young adults with signs/symptoms resembling the childhood disease and characterized by minor corneal involvement [3]. VKC inflammation is variable (mild, moderate, or severe), ranging from seasonal (acute) to chronic, and resembling the perennial seasonal conjunctivitis [2].
Current knowledge indicates that several inflammatory and tissue remodeling factors contribute to signs and symptoms of VKC [4]. Infiltrating Th2 cells and eosinophils, recruited mast cells and activated fibroblast/myofibroblasts drive the chronic inflammatory process by releasing soluble mediators, cytokines/chemokines, adhesion molecules, neuropeptides, and growth factors [5-9]. Secreted proteins accumulate in the tear fluid, representing a "tear-print" of the inflamed ocular surface and a view to physiopathological status. Increased cytokines belonging to Th1 (IFN $\gamma$, IL2) and Th2 (IL4, IL5) subgroups have been detected in tears and conjunctival impression cytologies (IC) from VKC patients [7-10]. In addition, chemokines/adhesion molecules, growth/angiogenic/profibrogenic factors, and receptors of innate immunity contribute actively to the local inflammation, as observed in both tears and ICs, or provided by in vitro studies [11-13]. Advances in protein analysis have been done 
to provide new high-throughput technological methods to identify several proteins at once in biological samples, including tears and conjunctival specimens obtained according to the IC technique $[14,15]$.

To date, different clinical features and therapeutic outcome in VKC suggest the need for predictive approaches, the crosstalk between a new grading approach and suitable biomarkers [15-19]. Therefore, the aim of this study was to provide a comprehensive protein expression profile in tears from active and quiescent VKC, by using a tear-chip array coupled analysis of 60 different factors (herein referred to as candidates). The final attempt will be to identify some candidates (laboratory biomarkers) associated with VKC activity, in order to identify those candidates, of prognostic value, linked to a potential background allowing VKC reactivation.

\section{Materials and Methods}

2.1. Ethical Statement and Reagent/Plastic-Ware Information. Authorization to carry out the study was provided by the intramural Ethical Committee at the University Hospital Campus Bio-Medico (Rome, Italy). The approval included patient management, tear sampling, and full experimental procedures. Furthermore, all procedures of handling human samples were conducted in accordance with guidelines established by the Association for Research in Vision and Ophthalmology and adhered to the tenets of the Declaration of Helsinki with respect to human subjects.

Unless specified in the text, sterile plastic-ware and analytical grade reagents were from NUNC (Roskilde, Denmark), SERVA (Heidelberg, Germany), ICN (Costa Mesa, CA), Euroclone (Milan, Italy), and Sigma-Aldrich (St. Louis, MA, USA). Ultrapure MilliQ-Grade water was daily provided (DirectQ5 Millipore, Vimodrone, Italy).

2.2. Study Population and Tear Collection. After an accurate explanation of the study design and the description of the potential information arising from the study, the participants (patients and controls or their parents) provided written informed consent to proceed to tear sampling.

A total of 16 consecutive patients suffering from VKC $(15 \mathrm{M} / 1 \mathrm{~F}$; mean age $15.50 \pm 3.16 \mathrm{yrs}$, ranging from 8 to $20 \mathrm{yrs})$ were included in the study (all referring spontaneously to our Clinical Unit). Enrollment criteria included a positive history of VKC and the absence of topical/systemic therapy. VKC diagnosis was based on ocular surface inflammation characterized by itching, photophobia, tearing/mucous discharge, the presence of a giant papillary reaction on the upper tarsal conjunctiva, and/or at the limbus associated with the presence of eosinophils in conjunctival scrapings [4]. Exclusion criteria included the presence of coexisting ocular and/or ocular surface diseases; the presence of systemic diseases other than coexisting allergic rhinitis, asthma, or atopic dermatitis; use of contact lens; use of topical/systemic medications at the time of the sample collection. Itching, tearing, photophobia, and foreign body sensation symptoms as well as the conjunctival hyperemia, mucous discharge, papillae, and corneal epithelial defects signs were scored from 0 to 3 (0: absent, 1: weak, 2: mild, and 3: severe). Finally, patients were graded according to the following severity score: 0 -quiescent, absence of ocular symptoms; 1 -mild, presence of ocular symptoms but not photophobia; 2-moderate, presence of symptoms and photophobia; 3-severe, presence of ocular symptoms and mild to moderate superficial punctuate keratitis (SPK); 4-very severe, presence of diffuse superficial keratopathy and/or corneal ulcer [4].

Ten sex/age matched healthy control volunteers with no signs/symptoms of conjunctivitis or ocular surface disease and not receiving systemic/local medications (steroids and/or antiallergic drugs or eye-drop tear substitute or surgical treatment) were enrolled for appropriate comparisons ( $5 \mathrm{M} / 5 \mathrm{~F}$; mean age $13.50 \pm 7.56 \mathrm{yrs}$, ranging from 8 to $29 \mathrm{yrs}$ ).

At the end of ophthalmic examination, the subjects underwent nonanesthetized tear collection. Briefly, tears were sampled according to the standardized "eye-flush" procedure implying the addition of $50 \mu \mathrm{L}$ sterile Balanced Salt Solution (BSS; Alcon Laboratories Inc., Fort Worth, TX) and a quick collection of tears with sterile single-wrapped plastic micropipettes (Sigma) [20-22]. The eye-flush procedure is consistent with the choice to collect tears in an extreme suitable/comfortable way for this "younger and anxious" study population [20,21]. All tear samples were quickly supplemented with protease inhibitors (Pierce Biotechnology, Rockford, IL) and stored at $-20^{\circ} \mathrm{C}$. Delivery from Clinical Unit to the Laboratory Unit was performed using an isothermal cage (CryoCooler; Starlab Intl GmbH, Ahrensburg, Germany), avoiding temperature changes, according to national rules and standardized operating procedures.

2.3. Protein Extraction, Quantification, and Electrophoresis. Tear samples were diluted in cold lysis buffer (50 mM Tris$\mathrm{HCl}, 150 \mathrm{mM} \mathrm{NaCl}, 1 \mathrm{mM}$ EDTA, 0.1\% Nonidet P-40, $1 \mathrm{mM}$ $\mathrm{NaF}$, and $1 \mathrm{mM}$ PMSF; pH 7.5), briefly sonicated (VibraCell; Sonics, Newtown, CT), and clarified by centrifugation (13000 rpm/7 min). For protein quantification, $3 \mu \mathrm{L}$ samples were analyzed with the A280 program (Nanodrop; Celbio, Milan, Italy). Tear samples showing low protein amounts were from patients/subjects having symptoms of ocular dryness and reduced mucus production. Protein separation was performed under reducing conditions on $4-12 \%$ mini-SDS polyacrylamide gels (SDS-PAGE; $150 \mathrm{~V} /$ frontline; Miniprotean3 apparatus; Biorad, Hercules, CA, USA) and the bands were transferred to Hybond membranes (GE Healthcare, Buckinghamshire, UK) under semidry conditions (13 V/25 min; semidry transblot system; Biorad). Prestained broad weight marker was run in parallel (10$250 \mathrm{kDa}$ prestained marker; Biorad). Bands were visualized by Ponceau S. Samples having high albumin/IgGs protein profiles were cleared (GE Healthcare).

2.4. Chip-Based Protein Arrays. Protein analysis was performed on chip-based arrays provided on glass slides, each comprising 14 identical subarrays containing 60 factors (antibody spots in duplicate) retrospectively selected (literature search) for custom-built array chips (Ray Biotech, Norcross, CA). Briefly, normalized protein extracts $(350 \mathrm{ng} / \mathrm{mL}$; $70 \mu \mathrm{L} /$ well) were diluted and hybridized in subarrays. $\mathrm{VKC/control} \mathrm{samples} \mathrm{were} \mathrm{processed} \mathrm{simultaneously} \mathrm{and} \mathrm{the}$ 
procedures of sample dilution, incubation/washing, detection, and labeling were according to the manufacturer's recommendation. Spin-dried slides were scanned in a GenePix 4400 Microarray platform (Molecular Devices LLC, Sunnyvale, Silicon Valley, CA). The specific area (grid; array/spot) was first manually spotted and then automatically adjusted by the software, and the capturing conditions were routinely applied to all glass slides. Images were uniformly adjusted for size, brightness, contrast, and chip-to-chip comparisons by the software, and provided as 8-bit Tiff converted format (Axon GenePix Pro 6.0 software; Molecular Devices). Interassay normalization was guaranteed by the presence of multiple internal controls for each subarray. The minimum sensitivity range of the array was $3.8-56 \mathrm{pg} / \mathrm{mL}$.

2.5. Statistical Analysis. The pooled-samples choice was not considered in this cohort study, guaranteeing good statistical power and biological sensitivity/variance. The Fluorescent Intensity (FI) values (spot) were obtained by subtracting the background signal (GenePix Pro 6.0 software). Single FI values were entered into a Microsoft Excel database (Microsoft, Redmond, WA) and duplicate spots outside the $10 \%$ coefficient of variability were refused from the statistical analysis. FDR value of 0.01 was set. FI averages (means \pm SD) were automatically calculated from replicates (2 spots) of not-pooled tear samples. Comparisons between VKC and control groups or within VKC groups were performed by using the two-sided unpaired $t$-test analysis (SPSS ver. 15; IBM Inc., Chicago, IL). As cut-offs, $\geq 2$-fold changes (FC; herein defining the abundance in a given candidate (factor) with respect to control) and $p \leq 0.05$ or $p<0.00083(0.05 p$ value/ 60 targets) for multiple testing with the Bonferroni correction were considered. Correlations between differentially expressed candidates and clinical findings were calculated by Spearman correlation rank test (rho $\geq 0.5$ and $p \leq 0.05$ or $p<0.00083)$. Volcano plots and Venn diagrams were used to illustrate microarray data sets and results $[23,24]$.

\section{Results}

The entire study population included 16 consecutive VKC patients and 10 normal subjects (see M\&M). The clinical and biochemical data of VKC patient group and subgroups are summarized in Table 1. Briefly, nine out of 16 patients referred to our Clinical Unit out-of-season, having no symptoms of disease (quiescent VKC) and 7 out of 16 patients referred to our Clinical Unit in-season, showing signs and symptoms of disease (active VKC). The Total Symptom Score (TSyS) and the Total Sign Score (TSS) were, respectively, $7.33 \pm 4.51$ and $8.67 \pm 3.05( \pm \mathrm{SD})$. The mean overall scores were $0-1$ in quiescent and 3-4 in active groups. A 1.27-fold increase of total protein concentration was quantified in quiescent samples while a 2.83 -fold increase was detected in active VKC samples ( $p<0.05$, as compared to controls). Although a trend toward a decrease in total protein amount was detected in tears from the left eye, with respect to right one $(p>0.05)$, no significant intragroup changes were observed between active and quiescent total protein amounts. A representative total protein sketch is depicted in Figure 1, showing

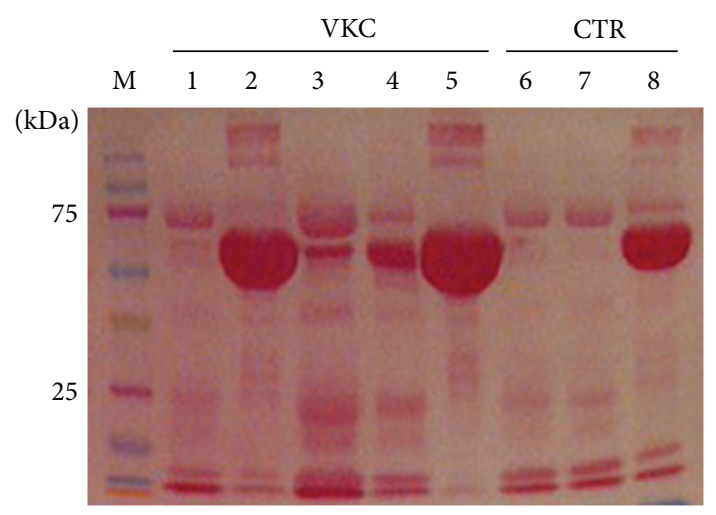

FIGURE 1: Tear protein profile. Equal protein amounts ( $20 \mu \mathrm{g} / \mathrm{lane} /$ sample) were subjected to electrophoretic separation (SDS-PAGE) and membranes were stained with Ponceau $S$ before image acquisition (see M\&M). Note the presence of albumin $(60 \mathrm{kDa})$, low/high IgG bands $(40 / 100 \mathrm{kDa})$, and fibronectin $(200 \mathrm{kDa})$ in some tear samples (1-5, VKC; 6-8 healthy controls). To retrieve low-expressed antigens, samples showing high albumin/IgGs were treated with a specific preclearing kit (see M\&M).

TABLE 1: Study population: overall description of some demographic and biochemical data of quiescent and active subgroups.

\begin{tabular}{|c|c|c|}
\hline Patient group & \multicolumn{2}{|c|}{ VKC } \\
\hline Number $(M / F)$ & \multicolumn{2}{|c|}{$16(15 / 1)$} \\
\hline Therapy (topical/systemic) & \multicolumn{2}{|c|}{ none } \\
\hline Patient subgroups & Quiescent & Active \\
\hline Number & 9 & 7 \\
\hline Seasonal gap & outside & inside \\
\hline Mean overall score & $0-1$ & $3-4$ \\
\hline Tear protein content $(\text { mean } \pm S D)^{\S}$ & $3.67 \pm 0.84$ & $8.19 \pm 1.56$ \\
\hline
\end{tabular}

${ }^{\S}$ Tears were sampled with the eye-flush technique and total proteins were measured according to the A280 Nanodrop program (see M\&M). Total protein concentration in control tear samples: $2.89 \pm 0.56$. Total protein values are expressed in $\mu \mathrm{g} / \mu \mathrm{L}$.

normalized VKC and control tear extracts resolved in a SDSPAGE.

To recognize VKC proteins of prognostic value in a whole array of potential candidates, both quiescent and active VKC tears were subject to a chip-based protein array evaluation and appropriate statistical analysis. Proteins were selected from a literature search and antibodies were thereafter assembled. Both quiescent and active VKC tear samples were hybridized on chip-based arrays followed by a case-control statistical analysis. As shown in Figures 2(a)-2(b), an overall increased fluorescence was observed in VKC arrays (a), although few spots were also positive in controls (b). Protein expression profiles were similar in tears from left and right eyes. The whole array map is displayed in Table 2.

From this active VKC versus control comparison, 4 out of 16 candidates showed $\mathrm{a} \geq 4.00$-fold $(p<0.0001)$ differential expression (NT4, TACE, and TNF $\alpha$ converting enzyme allowing the cleavage of soluble form of TNF $\alpha$ receptor and Macrophage Inflammatory Protein (MIP) $1 \alpha$ and $3 \alpha$ ); 16 out of 60 candidates had $\mathrm{a} \geq 3.00$-fold $(p<0.005)$ differential 


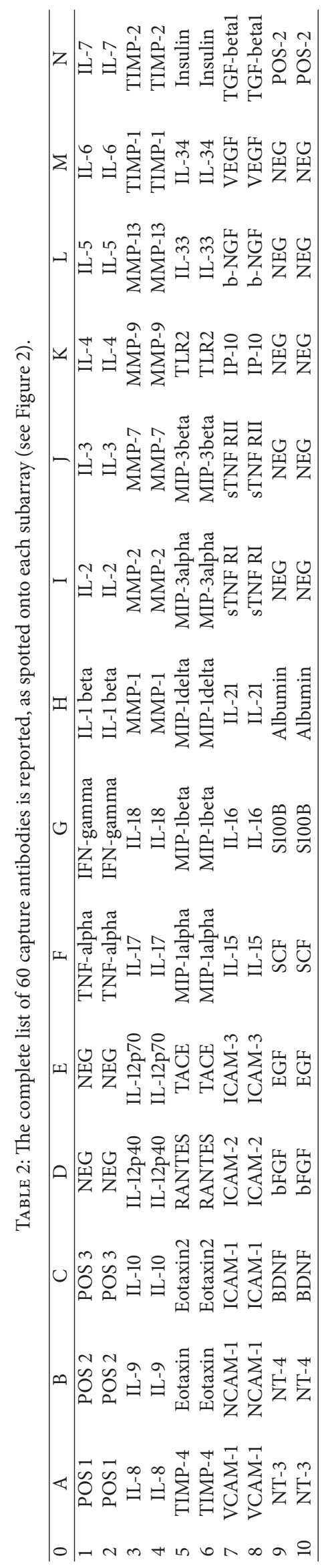




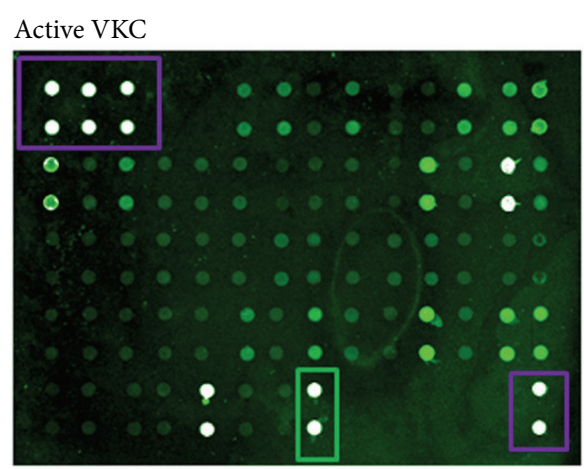

(a)

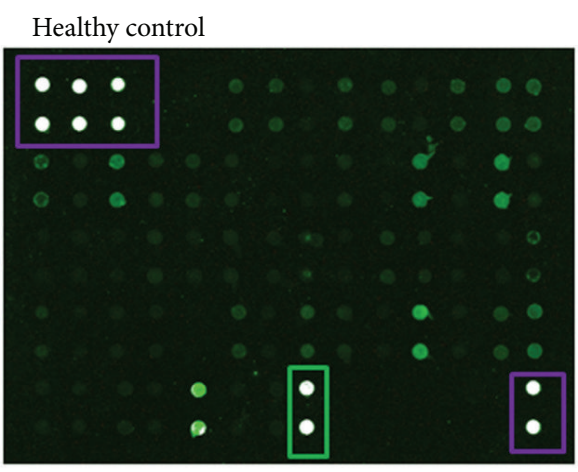

(b)

FIGURE 2: Overview of the protein array. Equal protein amounts were loaded for each subarray and the presence of an equal number of VKC: control samples were guaranteed for each array-chip (14 subarrays). (a), (b) Representative active VKC (a) and control (b) arrays, as provided by the GenePix scanner (with no color adjustment). White spots framed violet are positive controls, and black spots are negative referring controls and black spots framed green are albumin specific signals.

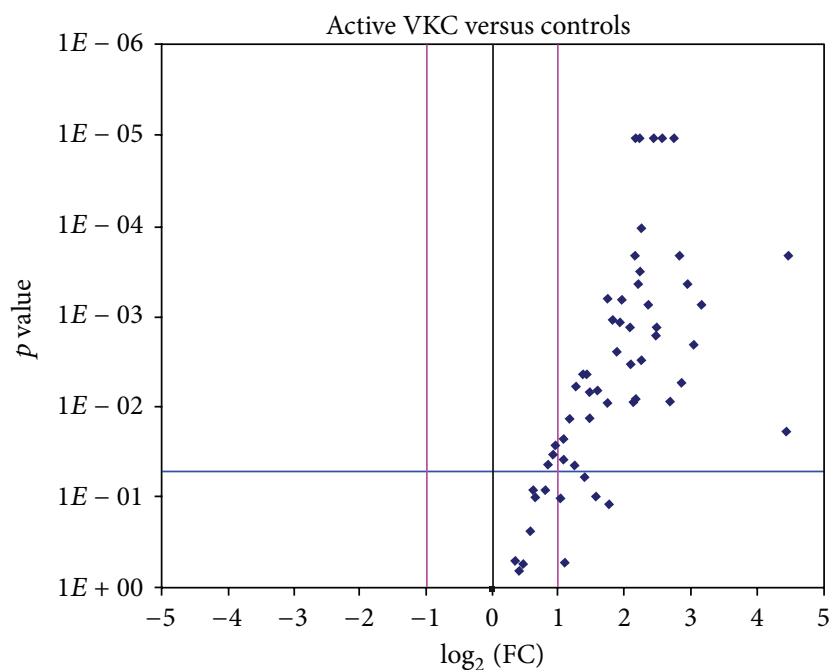

(a)

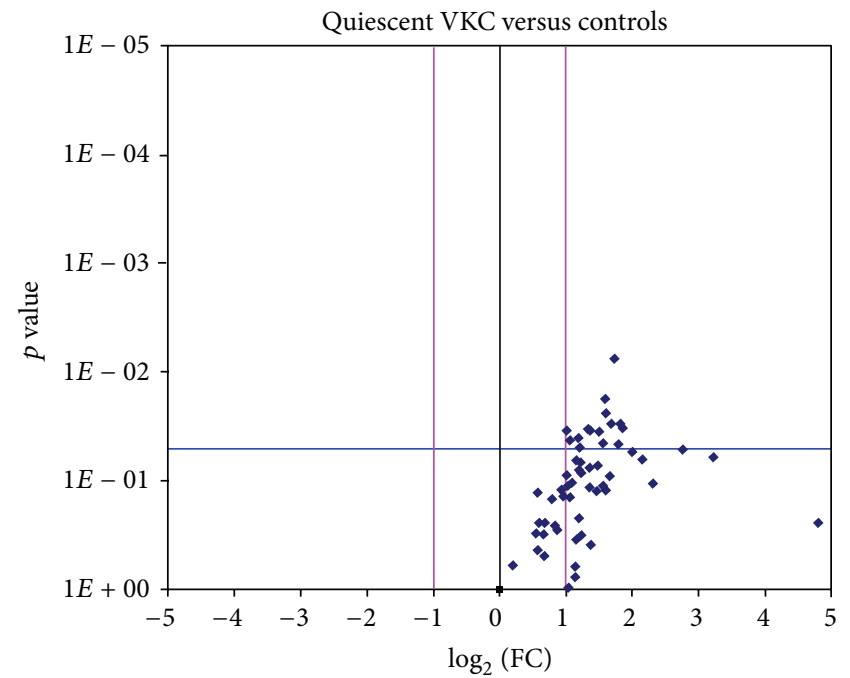

(b)

FIgURE 3: Plot graphs of the tear expression profile in active and quiescent VKC. Fold changes $\left(\log _{2}\right.$ (FC); $x$-axis) are ranked in Volcano plots according to the statistical significance ( $p$ values as negative $\log _{10} ; y$-axis). For each marker, FC between case and control values were calculated from mean of Fluorescent Intensity values provided by the software, as described in M\&M. The two-sided unpaired $t$-test comparisons of active (a) and quiescent (b) samples were carried out versus controls. Both $\pm 2 \mathrm{FC}$ and $p \leq 0.05$ were used as initial cut-offs. Red lines indicate differences of \pm 1 FC $\left(\log _{2}\right)$ and blue line shows the initial significance level. Those candidates, having $\geq 2$ FC and $p \leq 0.05$ initial cut-offs, are localized in the upper left quadrant.

expression (cytokines IL1 $\beta$, IL2, IL8, IL9, IL15, IL17, and IL21, growth factors NT4, BDNF, $\beta$ FGF, and SCF, adhesion molecules TACE, MIP1 $\alpha$, MIP $3 \alpha$, and ICAM $2 / 3$, and soluble receptors sTNFRI/II) and 8 out of 16 candidates showed $\mathrm{a} \geq 3.00$-fold $(p<0.01)$ differential expression (IL4/5, IL12p40, IL16, IL17, IL18, and IL33/34). With respect to the expression of enzymes involved in the ECM metabolism, the MMP1 (5.81-fold with $p<0.0001$ ) and MMP13 (5.36-fold; $p<0.0001)$, the MMP2 (3.49-fold; $p<0.005)$, and the MMP7 (6.35-fold; $p<0.01$ ) were highly increased in active VKC tears, with respect to controls. While TIMP1/2 tissue inhibitors were not increased in active subgroup, the tissue inhibitor TIMP4 showed a significant increase in active VKC tear samples (4.44-fold; $p<0.01$ ), as compared to controls. The Volcano plot underlying the complete protein expression in active VKC tears is shown in Figure 3(a).

From the quiescent VKC versus control comparison, some candidates were also increased in quiescent VKC tears (IL1 $\beta$, IL15, IL21, $\beta$ NGF, NT4, BDNF, $\beta$ FGF, SCF, MMP1/2, Eotaxin2, TACE, MIP1 $\delta$, MIP $3 \alpha$, NCAM1, and ICAM2; $\geq 2.00$-fold and $p<0.05$, versus controls). MMP1 and MMP2 were found slightly increased in quiescent VKC tears (resp., 2.90- and 2.06-fold; $p<0.05$ ). As above, a 3.94-fold increase was detected for TIMP4, although slightly significant $(p=0.051)$. Finally, IL33 expression was particularly high in both active (7.18-fold; $p<0.05)$ and quiescent (4.89-fold; 


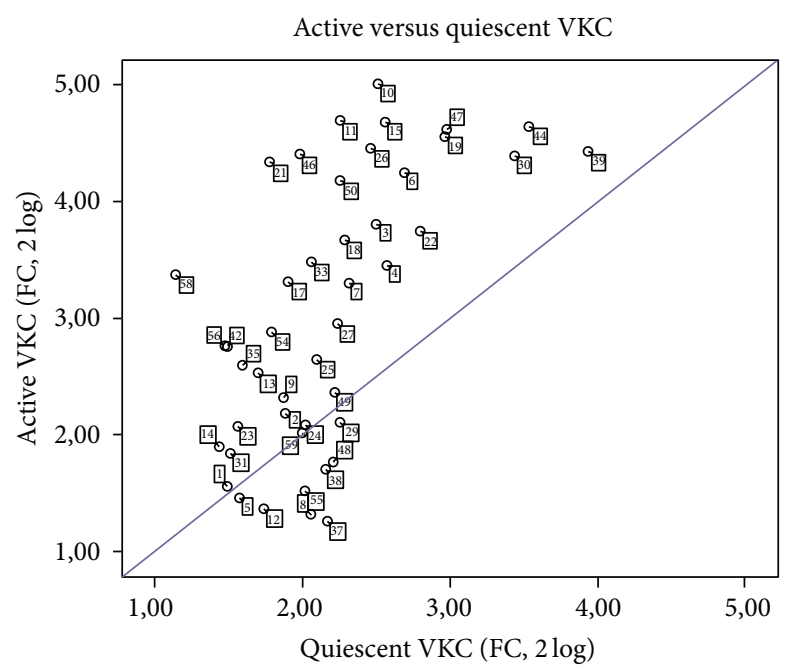

(a)

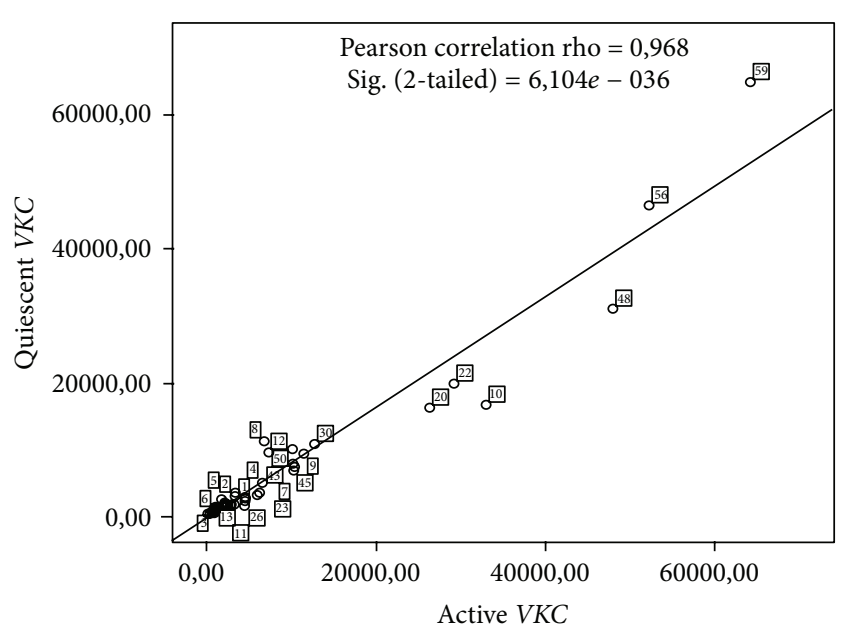

(b)

FIGURE 4: Comparison between quiescent and active subgroups. The scatter plot in (a) shows the candidate fold changes between quiescent and active tears, as calculated from mean of FI values provided by the software (see M\&M). Those candidates, having $\geq 2 \mathrm{FC}$ and $p \leq 0.05$ initial cut-offs, are localized in the lower left quadrant. Correlation between quiescent and active biomarkers in VKC tears is shown in MFI values (b). The Pearson correlation analysis is reported in the panel. Note the close association of quiescent and active VKC candidates in the lower left region of the slope.

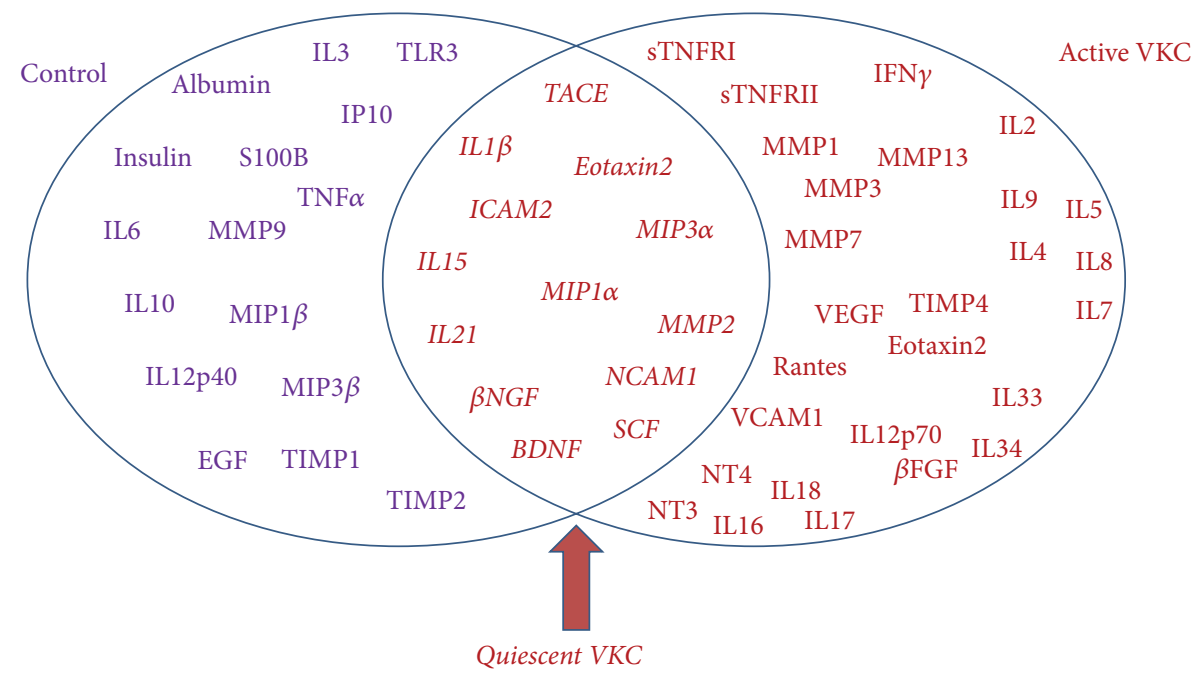

FIGURE 5: Venn diagram of predicted candidate biomarkers. Venn diagram showing the partial overlap between quiescent and active VKC groups. As predicted by this experimental approach, overlapping biomarkers are highlighted by the red arrow. At least in this study, all candidates showed at least $\geq 2$-fold differences and a $p$ value $\leq 0.05$ or 0.00085 , according to the Bonferroni correction.

$p>0.05)$ VKC tears, with respect to controls. The Volcano plot displaying the complete protein expression in quiescent VKC tears is shown, respectively, in Figure 3(b).

The whole distribution of quiescent/active candidate fold changes in tear is shown in Figure 4(a) ( $\log _{2}$ expression). The scatter plot indicates that the majority of factors common to both VKC states are displayed in the lower region (low fold expression). Those factors highly expressed are mainly of active tear root (see upper quadrant in Figure 4(a)). The linear regression highlights the correlation between active and quiescent VKC tears (Pearson correlation rho $=0.968$, $p=6.104 e-036 ;$ Figure 4(b)). As summarized in Figure 5, 16 out of 60 candidates overlap active and quiescent VKC states and include the following: IL1 $\beta$, IL15, IL21 (inflammatory cytokines), $\beta$ NGF, NT4, BDNF, SCF (growth factors), MMP1, MMP2 (tissue proteases) and Eotaxin2, TACE, MIP1 $\alpha$, MIP3 $\alpha$, NCAM1, and ICAM2 (chemokines and adhesion molecules). A further analysis of quiescent versus active VKC indicated that three out of those 16 candidates (NT4, $\beta F G F$, and MMP1; see bold font in Table 3) were significantly expressed in the active form with respect to the quiescent group $(p<0.05)$. Albeit not significant, MIP1 $\beta$ was extremely 
TABLE 3: Summary of the protein profile expression in all subgroups. The 16 differentially expressed proteins (candidate biomarkers) are functionally grouped in specific clusters: cytokines (Th1-Th2-Th9-Th17 subtypes), growth factors (neurotrophins and fibrogenic/angiogenic factors), chemokines/adhesion molecules, tissue proteases (specific ECM enzymes/inhibitors), and other molecules (soluble receptors and referring proteins). Column 2 provides the significance (Sig.) for active and quiescent biomarkers, as obtained with respect to controls $\left({ }^{*} p<\right.$ $0.05 ;{ }^{* *} p<0.01{ }^{* * *} p<0.005 ;{ }^{* * * *} p<0.0001$; two-sided unpaired $t$-test analysis). The related quiescent: active comparisons (fold changes $(\mathrm{FC})), p$ values and significances are shown. Bold font indicates candidates common to quiescent and active VKC $(p>0.05)$. The last column indicates each candidate incthe related VKC literature and the $\$$ symbol highlights only those papers concerning VKC tissues.

\begin{tabular}{|c|c|c|c|c|c|c|}
\hline \multirow{2}{*}{$\begin{array}{l}\text { Clusters } \\
\text { Candidate biomarkers }\end{array}$} & \multicolumn{2}{|c|}{ Sig., cases versus controls } & \multicolumn{3}{|c|}{ Active versus quiescent } & \multirow{2}{*}{$\begin{array}{c}\text { Literature } \\
\text { Ref }\end{array}$} \\
\hline & Quiescent & Active & FC & $p$ value & Sig. & \\
\hline \multicolumn{7}{|l|}{ Cytokines } \\
\hline $\operatorname{IL} 1 \beta$ & $*$ & $* * *$ & 1.52 & 0.2326 & & {$[6]$} \\
\hline IL15 & $*$ & $* * *$ & 1.83 & 0.0628 & & \\
\hline IL21 & $*$ & $* * *$ & 1.54 & 0.1714 & & \\
\hline \multicolumn{7}{|l|}{ Growth factors } \\
\hline$\beta \mathrm{NGF}$ & * & $* *$ & 1.34 & 0.3885 & & {$[13,40]^{\S}$} \\
\hline NT4 & $*$ & $* * * *$ & 1.81 & 0.0410 & $*$ & \\
\hline $\mathrm{BDNF}$ & $*$ & $* * *$ & 1.33 & 0.3580 & & {$[41]$} \\
\hline$\beta \mathrm{FGF}$ & * & $* * *$ & 2.24 & 0.0286 & * & {$[41]$} \\
\hline SCF & $*$ & $* * *$ & 1.28 & 0.4784 & & {$[41]$} \\
\hline \multicolumn{7}{|l|}{ Tissue proteases } \\
\hline MMP1 & * & $* * * *$ & 2.01 & 0.0417 & * & {$[41]$} \\
\hline MMP2 & $*$ & $* * *$ & 1.69 & 0.0764 & & {$[41]$} \\
\hline Eotaxin2 & $*$ & $*$ & 3.19 & 0.1132 & & {$[6,34,42]$} \\
\hline TACE & $*$ & $* * * *$ & 1.95 & 0.0554 & & \\
\hline MIP1 $\alpha$ & $*$ & $* *$ & 1.32 & 0.4775 & & \\
\hline MIP3 $\alpha$ & $*$ & $* * * *$ & 1.55 & 0.1446 & & \\
\hline NCAM1 & $*$ & $* *$ & 1.85 & 0.0789 & & \\
\hline ICAM2 & $* *$ & $* * *$ & 2.34 & 0.0226 & & [34] \\
\hline
\end{tabular}

Two-sided unpaired $t$-test analysis (fold changes (FC), $p$ values, and case/control significance $\left(\right.$ Sig.; $\left.{ }^{*} p<0.05 ;{ }^{* *} p<0.01 ;{ }^{* * *} p<0.005 ;{ }^{* * *} p<0.0001\right)$ ) (see M\&M).

high in both active and quiescent specimens (resp., 32.86-fold and 27.61-fold; both $p>0.05$ versus controls).

\section{Discussion}

Currently in force for a wide-range biomolecular investigation of disease-linked profiles, the microarray platform represents an excellent high-throughput technology that facilitates the simultaneous detection of more than a few "biomarker or gene/oligo/protein candidates" [14, 15, 25]. By using the protein-based approach set up in fluorescence, we addressed the question as to whether some protein candidates (prospective indicators of inflammation and/or tissue remodeling) might be expressed in both active and quiescent VKC tears, representing pointer of disease activity and useful prognostic factors in the clinical practice. As discussed below, we confirmed some protein candidates and identified new ones in active $\mathrm{VKC}$ and recognized some common to both quiescent and active forms. These acute and quiescent overlapping candidates are IL1 $\beta$, IL15, IL21, $\beta N G F, B D N F$, SCF, MMP2, Eotaxin2, TACE, MIP1 $\alpha$, MIP $3 \alpha$, NCAM1, and ICAM2, as shown in the Venn diagram in Figure 5 (pointed by red arrow).
Firstly, the widespread protein expression in active VKC with respect to control tears is in line with the inflammatory and tissue remodeling process occurring at the ocular surface. Cytokines, chemokines, and adhesion molecules, growth factors, and some soluble receptors play a major role in chronic inflammatory disorders, through a tidy regulation of cell influx leading to an exacerbation of the local inflammation and the promotion of corneal lesions [3]. The observation of the increased expression of IL1 $\beta$, IL15, IL21, Eotaxin2, TACE, MIP1 $\alpha$, MIP $3 \alpha$, NCAM1, ICAM2, $\beta$ NGF, NT4, BDNF, $\beta$ FGF, SCF, MMP1, MMP2, and sTNFRI/II is in line with literature, as reported in previous studies carried out on VKC tears, ICs, and conjunctival biopsies [5-13]. Particularly, IL9 has been associated with seasonal-mediated allergic activation $[26,27]$; IL7, IL15, and IL21 have been reported in chronic inflammatory and tissue remodeling process [27, 28]; IL17 has been detected only in VKC sera [27, 29]; IL18 has been implicated in Th1/Th2-response modulation [30], and IL16 has been correlated with IgE level expression [31]. The unchanged TNF $\alpha$, IL6, and IL10 expression and slight IFN $\gamma$ increase in active VKC might be consistent with the absence of corneal involvement and tissue remodeling, as provided by the VKC score $[12,27,32]$. The high expression of IL33 and 
IL34 in active VKC might be linked to the Th2-response and the IgE dependent activity, as well as to the release of Th2derived cytokines through a stimulatory effect on mast cells, eosinophils, and basophils [33].

Although at a relatively low level, the common expression of some candidates in quiescent VKC tears would imply that these factors might provide long-lasting conditions suitable for disease reactivation and therefore might be proposed as a potential indicator of forthcoming reactivation. Of those cytokines significantly expressed in active VKC tears (IL3, IL7, IL9, IL15, IL16, IL17, IL18, IL21, and IL33/34), only IL1 $\beta$, IL15, and IL21 were monitored in quiescent VKC. Overexpressed in active VKC, Eotaxin2 detection in quiescent tears might be indicative of a forthcoming eosinophil recruitment, according to in vitro/in vivo chemoattractant studies [34]. In addition, MIP1 $\alpha$, MIP3 $\alpha$, TACE, NCAM1, and ICAM2 might represent new potential candidates for their well-known cell homing effects [34-37]. It is noteworthy that MIP1 $\alpha$-MIP3 $\alpha$ has been recently implicated in the epithelial cell activation as well as eosinophil, lymphocyte, and neutrophil tissuehoming and, more appropriately, MIP $3 \alpha$ has been recently prospected as a serum prognostic factor at least for systemic inflammatory diseases [37]. NCAM1 and ICAM2 expression in quiescent VKC tears might be suggestive of a sustained local Th2-driven response as well as neutrophils, NK cell, eosinophil, and mast cell noiseless activity [10, 12, 34-37]. Finally, the common expression of TACE in both active and quiescent VKC tears might be indicative of a potential synthesis/release of IL8, a potent regulator of neutrophil activity [36].

A cross-talk between neuronal and nonneuronal cells has been described in VKC and a bidirectional (neuro)protective and/or anti-inflammatory cross-talk between immune and structural cells might be prospected under quiescent conditions $[13,38,39]$. While NGF, BDNF, NT3/4, SCF, VEGF, and TGF $\beta 1$ changes in active VKC might be due to production by activated eosinophil, mast cell, and T cells $[40,41]$, the observation of NGF, NT4, BDNF, and $\beta$ FGF expression in quiescent VKC is actually an open question $[41,42]$. Some neuroprotective effects might be proposed for NGF, NT3/4, and BDNF overexpression in quiescent VKC tears [39]. On the contrary, SCF expression in quiescent VKC tears might be explained with the active VKC microenvironment characterized by chemotactic/survival/regulatory activities of mast cell and eosinophils [43].

Several tissue proteases/inhibitors (MMPs/TIMPs) trigger long-lasting ECM metabolism in active VKC, while playing homeostatic effects under normal states $[1,2]$. The uncontrolled MMPs/TIMPs might significantly contribute to chronic-sustained extensive tissue remodeling and giant papillae formation, by means of collagen/basal membrane degradation and inflammatory cell transmigration, until the development of corneal erosions, as observed in severe VKC forms $[15,44]$. Except for MMP1/MMP2/MMP13 previously documented in VKC, the selective MMP7/TIMP4 overexpression in active VKC and the common expression of MMP2 in both quiescent and active forms represent new attractive findings. Apparently not in line with previous studies, the slight MMP9 expression in active VKC is consistent with the unchanged TNF $\alpha$ values (a major MMP9 modulator) and might be supported by the absence of superficial corneal involvement or ulcers in this study population [44]. As reported, the main MMP9 activity occurs at the corneal basement and correlates with papillae development and corneal erosion in VKC [44]. The significant increase of the regulatory MMP7 appears to be of great interest. MMP7 might allow the activation of other MMPs and the accessibility of several neurotrophic/angiogenic factors (VEGF, TGF $\beta 1$, and NGF) $[15,45]$. With respect to NGF-MMP7 interaction, the proNGF cleavage has been recently associated with some neuroprotective activities [45].

Overall, VKC is a self-limiting eye disease characterized by a chronic inflammatory process (mostly seasonal driven) and overt ECM remodeling [1-6]. None of the current therapies available can really protect from VKC discomfort and corneal hurt, suggesting that any attempts to offset the recurrent/long-standing inflammation (disease/complications) or foresee the seasonal activation/exacerbation are welcome from ophthalmologists [1618]. Since some tear proteins represent the output of cells populating the ocular surface (merely conjunctival epithelium/stroma) and since VKC prolongation is dependent on physical/biological stressors and microenvironment, the identification of some laboratory markers common to acute and quiescent VKC forms might represent a foremost target for developing alternative strategies to counteract VKC reactivation. Herein, a predictive VKC-tear protein profile might be hypothesized for IL1 $\beta$, IL15, IL21, Eotaxin2, TACE, MIP1 $\alpha$, MIP3 $\alpha$, NCAM1, ICAM2, $\beta$ NGF, NT4, BDNF, $\beta$ FGF, SCF, MMP1, and MMP2, all quantified in acute VKC and detected also in quiescent VKC tears. Moreover the eye-flush tear sampling technique appears appropriate as it allows an easy collection and a good volume (diluted tears) for the chipbased array. The specificity of this way of sampling is provided by the observation that only a few of the significantly changed proteins were identified.

Understanding the potential candidates might be helpful for prognostic purposes as well as for developing appropriate strategies to counteract VKC either outside or during the season, and with respect to other VKC-associated conditions (i.e., dry eye).

\section{Conflict of Interests}

All authors declare no conflict of interests and certify that they have no affiliations with or involvement in any organization or entity with any financial interest or nonfinancial interest in the subject matter or materials discussed in this paper.

\section{Authors' Contribution}

Alessandra Micera and Stefano Bonini conceived and designed the experiment. All authors performed the experiments. All authors analyzed the data. Alessandra Micera and Stefano Bonini contributed reagents/materials/analysis tools. All authors wrote the paper. 


\section{Acknowledgments}

This study was partially supported by the Italian Ministry of Health and the national 5xMille 2009 tax donation to IRCCSG. B. Bietti Foundation. The authors wish to express gratitude to Fondazione Roma for continuous intramural support. The authors thank Dr. Roberto Sacco (Laboratory of Molecular Psychiatry and Neurogenetics, UCBM, Rome, Italy) and Dr. Magdalena Cortes (IRCCS-G.B. Bietti Foundation, Rome, Italy) for critical reading of the paper.

\section{References}

[1] S. Bonini, S. Bonini, A. Lambiase et al., "Vernal keratoconjunctivitis revisited: a case series of 195 patients with long-term followup," Ophthalmology, vol. 107, no. 6, pp. 1157-1163, 2000.

[2] S. Bonini, A. Lambiase, R. Sgrulletta, and S. Bonini, "Allergic chronic inflammation of the ocular surface in vernal keratoconjunctivitis," Current Opinion in Allergy and Clinical Immunology, vol. 3, no. 5, pp. 381-387, 2003.

[3] A. Leonardi, D. Lazzarini, L. Motterle et al., "Vernal keratoconjunctivitis-like disease in adults," American Journal of Ophthalmology, vol. 155, no. 5, pp. 796-803, 2013.

[4] S. Bonini, M. Sacchetti, F. Mantelli, and A. Lambiase, "Clinical grading of vernal keratoconjunctivitis," Current Opinion in Allergy and Clinical Immunology, vol. 7, no. 5, pp. 436-441, 2007.

[5] E. Uchio, S. Y. Ono, Z. Ikezawa et al., "Tear levels of IFNgamma, interleukin (IL) 2, IL4 and IL5 in patients with vernal keratoconjunctivitis, atopic keratitis and allergic conjunctivitis," Clinical \& Experimental Allergy, vol. 30, no. 1, pp. 103-109, 2000.

[6] A. Leonardi, S. J. Curnow, H. Zhan, and V. L. Calder, "Multiple cytokines in human tear specimens in seasonal and chronic allergic eye disease and in conjunctival fibroblast cultures," Clinical and Experimental Allergy, vol. 36, no. 6, pp. 777-784, 2006.

[7] E. B. Cook, J. L. Stahl, L. Lowe et al., "Simultaneous measurement of six cytokines in a single sample of human tears using microparticle-based flow cytometry: allergics vs. non-allergics," Journal of Immunological Methods, vol. 254, no. 1-2, pp. 109-118, 2001.

[8] A. M. Abu El-Asrar, K. Geboes, S. Al-Kharashi, K. F. Tabbara, L. Missotten, and V. Desmet, "Adhesion molecules in vernal keratoconjunctivitis," British Journal of Ophthalmology, vol. 81, no. 12, pp. 1099-1106, 1997.

[9] A. Lambiase, S. Bonini, A. Micera et al., "Increased plasma levels of substance $\mathrm{P}$ in vernal keratoconjunctivitis," Investigative Ophthalmology and Visual Science, vol. 38, no. 10, pp. 2161-2164, 1997.

[10] A. Lambiase, E. M. Normando, L. Vitiello et al., "Natural killer cells in vernal keratoconjunctivitis," Molecular Vision, vol. 13, pp. 1562-1567, 2007.

[11] S. Bonini, A. Micera, A. Iovieno, A. Lambiase, and S. Bonini, "Expression of toll-like receptors in healthy and allergic conjunctiva," Ophthalmology, vol. 112, no. 9, pp. 1528-1549, 2005.

[12] A. Leonardi, I. A. Fregona, M. Plebani, A. G. Secchi, and V. L. Calder, "Th1- and Th2-type cytokines in chronic ocular allergy," Graefe's Archive for Clinical and Experimental Ophthalmology, vol. 244, no. 10, pp. 1240-1245, 2006.

[13] L. Motterle, Y. Diebold, A. Enríquez de Salamanca et al., "Altered expression of neurotransmitter receptors and neuromediators in vernal keratoconjunctivitis," Archives of Ophthalmology, vol. 124, no. 4, pp. 462-468, 2006.

[14] R. Sack, L. Conradi, A. Beaton, S. Sathe, N. McNamara, and A. Leonardi, "Antibody array characterization of inflammatory mediators in allergic and normal tears in the open and closed eye environments," Experimental Eye Research, vol. 85, no. 4, pp. 528-538, 2007.

[15] A. Leonardi, S. Sathe, M. Bortolotti, A. Beaton, and R. Sack, "Cytokines, matrix metalloproteases, angiogenic and growth factors in tears of normal subjects and vernal keratoconjunctivitis patients," Allergy, vol. 64, no. 5, pp. 710-717, 2009.

[16] A. Leonardi, "Allergy and allergic mediators in tears," Experimental Eye Research, vol. 117, pp. 106-117, 2013.

[17] M. R. Allansmith and R. N. Ross, "Ocular allergy and mast cell stabilizers," Survey of Ophthalmology, vol. 30, no. 4, pp. 229-244, 1986.

[18] F. Mantelli, M. S. Santos, T. Petitti et al., "Systematic review and meta-analysis of randomised clinical trials on topical treatments for vernal keratoconjunctivitis," British Journal of Ophthalmology, vol. 91, no. 12, pp. 1656-1661, 2007.

[19] A. Lambiase, S. Minchiotti, A. Leonardi et al., "Prospective, multicenter demographic and epidemiological study on vernal keratoconjunctivitis: a glimpse of ocular surface in Italian population," Ophthalmic Epidemiology, vol. 16, no. 1, pp. 38-41, 2009.

[20] A. Inic-Kanada, A. Nussbaumer, J. Montanaro et al., "Comparison of ophthalmic sponges and extraction buffers for quantifying cytokine profiles in tears using Luminex technology," Molecular Vision, vol. 18, pp. 2717-2725, 2012.

[21] E. Uchino, S. Sonoda, K. Nakao, and T. Sakamoto, "Alteration of tear cytokine balance by eye closure: analysis by multicytokine assay," Graefe's Archive for Clinical and Experimental Ophthalmology, vol. 244, no. 6, pp. 747-749, 2006.

[22] N. Li, N. Wang, J. Zheng et al., "Characterization of human tear proteome using multiple proteomic analysis techniques," Journal of Proteome Research, vol. 4, no. 6, pp. 2052-2061, 2005.

[23] X. Cui and G. A. Churchill, "Statistical tests for differential expression in cDNA microarray experiments," Genome Biology, vol. 4 , no. 4 , article $210,2003$.

[24] C. A. Churchill, "Using ANOVA to analyze microarray data," BioTechniques, vol. 37, no. 2, pp. 173-175, 2004.

[25] H.-B. Kim, C.-K. Kim, K. Iijima, T. Kobayashi, and H. Kita, "Protein microarray analysis in patients with asthma: elevation of the chemokine PARC/CCL18 in sputum," Chest, vol. 135, no. 2, pp. 295-302, 2009.

[26] M. A. Zaki, A. Micera, H. Muntasser et al., "Conjunctival expression of IL9: a role for IL9 in ocular allergy?" Allergy, vol. 68, p. 97, 2013.

[27] M. Oray and E. Toker, "Tear cytokine levels in vernal keratoconjunctivitis: the effect of topical $0.05 \%$ cyclosporine a therapy," Cornea, vol. 32, no. 8, pp. 1149-1154, 2013.

[28] R. Spolski and W. J. Leonard, "The Yin and Yang of interleukin 21 in allergy, autoimmunity and cancer," Current Opinion in Immunology, vol. 20, no. 3, pp. 295-301, 2008.

[29] A. M. Zicari, M. Nebbioso, A. Zicari et al., "Serum levels of IL17 in patients with vernal keratoconjunctivitis: a preliminary report," European Review for Medical and Pharmacological Sciences, vol. 17, no. 9, pp. 1242-1244, 2013.

[30] K. Nakanishi, T. Yoshimoto, H. Tsutsui, and H. Okamura, "Interleukin-18 regulates both Th1 and Th2 responses," Annual Review of Immunology, vol. 19, pp. 423-474, 2001. 
[31] K.-G. Wu, T.-H. Li, C.-J. Chen, H.-I. Cheng, and T.-Y. Wang, "Correlations of serum interleukin-16, total IgE, eosinophil cationic protein and total eosinophil counts with disease activity in children with atopic dermatitis," International Journal of Immunopathology and Pharmacology, vol. 24, no. 1, pp. 15-23, 2011.

[32] A. Leonardi, P. Brun, M. Tavolato, M. Plebani, G. Abatangelo, and A. G. Secchi, "Tumor necrosis factor-alpha (TNF-alpha) in seasonal allergic conjunctivitis and vernal keratoconjunctivitis," European Journal of Ophthalmology, vol. 13, no. 7, pp. 606-610, 2003.

[33] T. Pecaric-Petkovic, S. A. Didichenko, S. Kaempfer, N. Spiegl, and C. A. Dahinden, "Human basophils and eosinophils are the direct target leukocytes of the novel IL-1 family member IL-33," Blood, vol. 113, no. 7, pp. 1526-1534, 2009.

[34] A. Leonardi, P. J. Jose, H. Zhan, and V. L. Calder, "Tear and mucus eotaxin-1 and eotaxin-2 in allergic keratoconjunctivitis," Ophthalmology, vol. 110, no. 3, pp. 487-492, 2003.

[35] M. Mrugacz, B. Zelazowska, A. Bakunowicz-Lazarczyk, M. Kaczmarski, and J. Wysocka, "Elevated tear fluid levels of MIP$1 \alpha$ in patients with cystic fibrosis," Journal of Interferon and Cytokine Research, vol. 27, no. 6, pp. 491-495, 2007.

[36] R. A. Black, "Tumor necrosis factor- $\alpha$ converting enzyme," International Journal of Biochemistry and Cell Biology, vol. 34, no. 1, pp. 1-5, 2002.

[37] T. Iwata, K. Tanaka, Y. Inoue et al., "Macrophage inflammatory protein-3 alpha (MIP-3a) is a novel serum prognostic marker in patients with colorectal cancer," Journal of Surgical Oncology, vol. 107, no. 2, pp. 160-166, 2013.

[38] M. Scuri, L. Samsell, and G. Piedimonte, "The role of neurotrophins in inflammation and allergy," Inflammation and Allergy_Drug Targets, vol. 9, no. 3, pp. 173-180, 2010.

[39] A. Micera, A. Lambiase, L. Aloe, S. Bonini, F. Levi-Schaffer, and S. Bonini, "Nerve growth factor involvement in the visual system: implications in allergic and neurodegenerative diseases," Cytokine \& Growth Factor Reviews, vol. 15, no. 6, pp. 411-417, 2004.

[40] A. Lambiase, S. Bonini, S. Bonini et al., "Increased plasma levels of nerve growth factor in vernal keratoconjunctivitis and relationship to conjunctival mast cells," Investigative Ophthalmology and Visual Science, vol. 36, no. 10, pp. 2127-2132, 1995.

[41] A. Leonardi, P. Brun, M. Tavolato, G. Abatangelo, M. Plebani, and A. G. Secchi, "Growth factors and collagen distribution in vernal keratoconjunctivitis," Investigative Ophthalmology and Visual Science, vol. 41, no. 13, pp. 4175-4181, 2000.

[42] J. Shoji, N. Inada, and M. Sawa, "Antibody array-generated cytokine profiles of tears of patients with vernal keratoconjunctivitis or giant papillary conjunctivitis," Japanese Journal of Ophthalmology, vol. 50, no. 3, pp. 195-204, 2006.

[43] V. Dolgachev, M. Thomas, A. Berlin, and N. W. Lukacs, "Stem cell factor-mediated activation pathways promote murine eosinophil CCL6 production and survival," Journal of Leukocyte Biology, vol. 81, no. 4, pp. 1111-1119, 2007.

[44] A. Leonardi, P. Brun, G. Abatangelo, M. Plebani, and A. G. Secchi, "Tear levels and activity of matrix metalloproteinase (MMP)-1 and MMP-9 in vernal keratoconjunctivitis," Investigative Ophthalmology and Visual Science, vol. 44, no. 7, pp. 30523058, 2003.

[45] S. Dozier, G. P. Escobar, and M. L. Lindsey, "Matrix metalloproteinase (MMP)-7 activates MMP-8 but not MMP-13," Medicinal Chemistry, vol. 2, no. 5, pp. 523-526, 2006. 


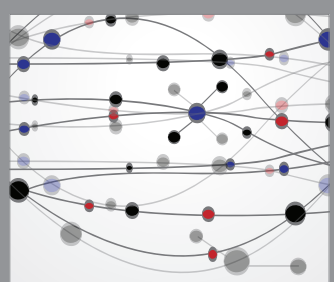

The Scientific World Journal
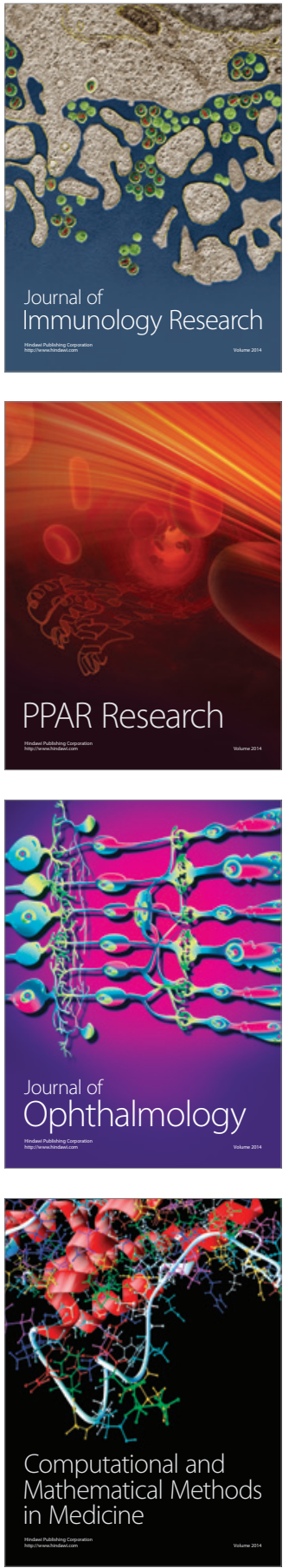

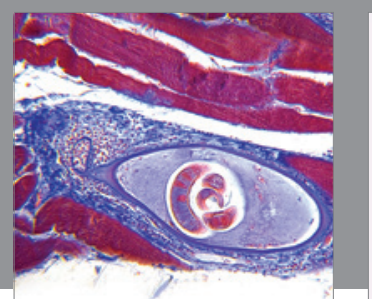

Gastroenterology Research and Practice

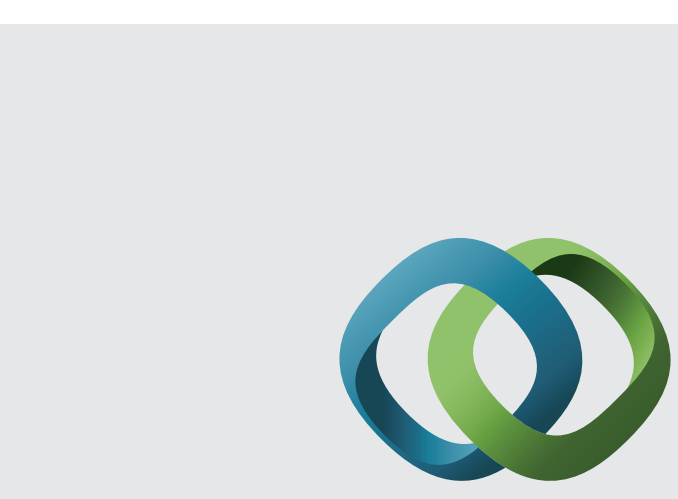

\section{Hindawi}

Submit your manuscripts at

http://www.hindawi.com
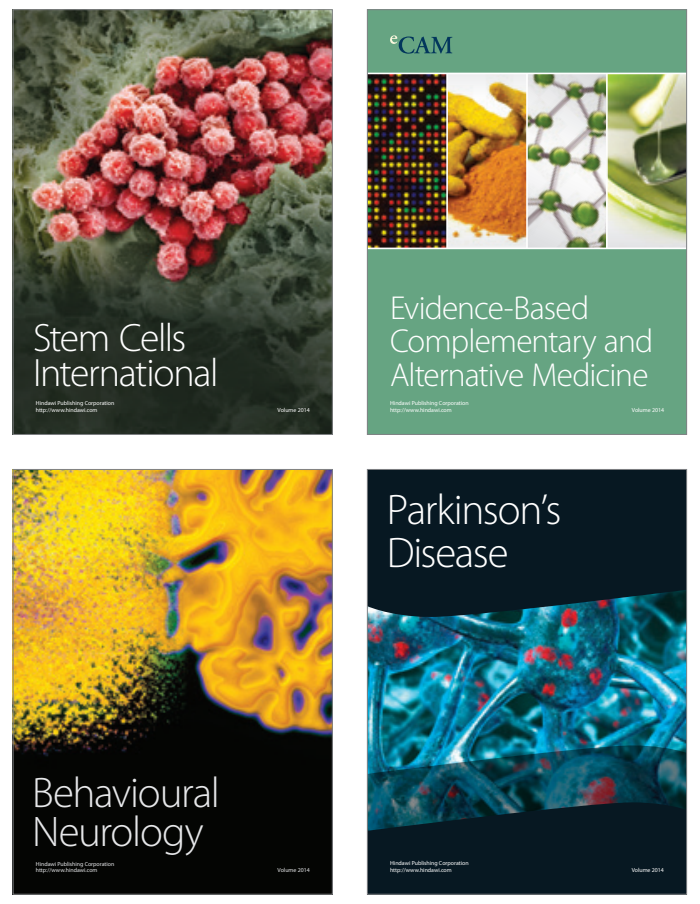
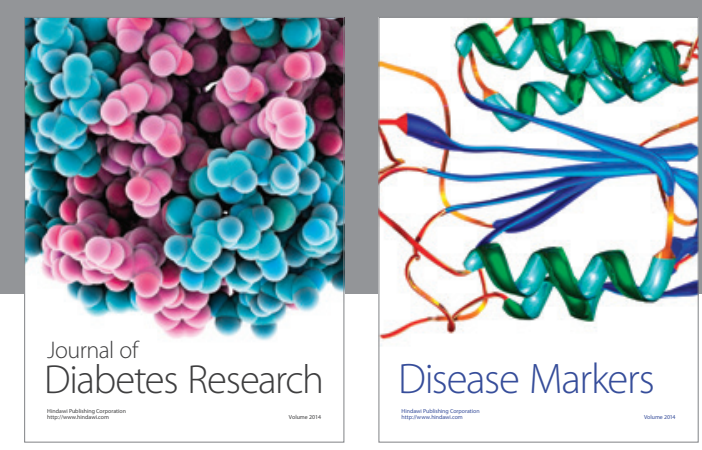

Disease Markers
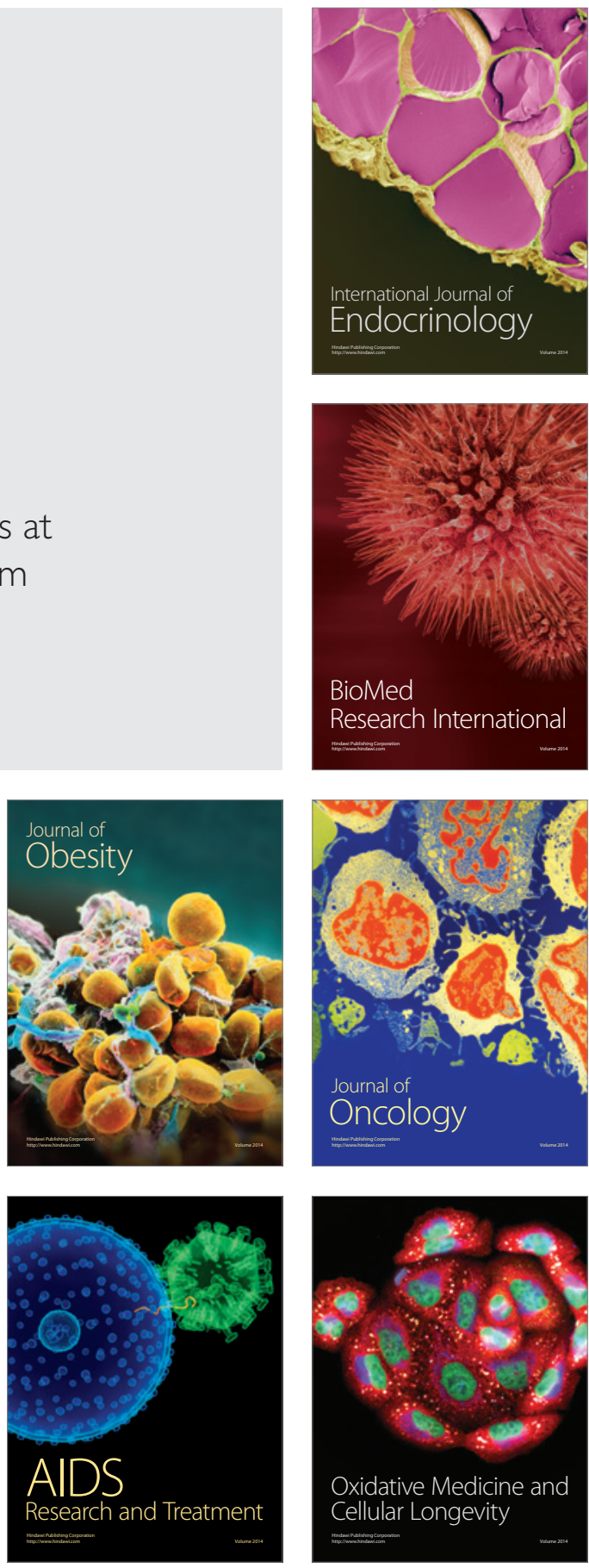